\title{
Antiangiogenic Effect of Bevacizumab: Application of Arterial Spin-Labeling Perfusion MR Imaging in a Rat Glioblastoma Model
}

\author{
(D)T.J. Yun, (DH.R. Cho, (D)S.H. Choi, (D) H. Kim, (D).-K. Won, (D).-W. Park, (D).-h. Kim, (D)C.-H. Sohn, and (D)M.H. Han
}

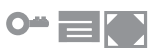

\begin{abstract}
BACKGROUND AND PURPOSE: The usefulness of arterial spin-labeling for the evaluation of the effect of the antiangiogenic therapy has not been elucidated. Our aim was to evaluate the antiangiogenic effect of bevacizumab in a rat glioblastoma model based on arterial spin-labeling perfusion MR imaging.
\end{abstract}

MATERIALS AND METHODS: DSC and arterial spin-labeling perfusion MR imaging were performed by using a 9.4T MR imaging scanner in nude rats with glioblastoma. Rats were randomly assigned to the following 3 groups: control, 3-day treatment, and 10-day treatment after bevacizumab injection. One-way analysis of variance with a post hoc test was used to compare perfusion parameters (eg, normalized CBV and normalized CBF from DSC MR imaging and normalized CBF based on arterial spin-labeling) with microvessel area on histology. The Pearson correlations between perfusion parameters and microvessel area were also determined.

RESULTS: All of the normalized CBV from DSC, normalized CBF from DSC, normalized CBF from arterial spin-labeling, and microvessel area values showed significant decrease after treatment $(P<.001, P<.001, P=.005$, and $P<.001$, respectively). In addition, normalized $C B V$ and normalized CBF from DSC and normalized CBF from arterial spin-labeling strongly correlated with microvessel area (correlation coefficient, $r=0.911,0.869$, and 0.860 , respectively; $P<.001$ for all).

CONCLUSIONS: Normalized CBF based on arterial spin-labeling and normalized CBV and normalized CBF based on DSC have the potential for evaluating the effect of antiangiogenic therapy on glioblastomas treated with bevacizumab, with a strong correlation with microvessel area.

ABBREVIATIONS: $\mathrm{ASL}=$ arterial spin-labeling; $\mathrm{GBM}=$ glioblastoma; $\mathrm{MVA}=$ microvessel area; $\mathrm{nCBF}=$ normalized $\mathrm{CBF} ; \mathrm{nCBV}=$ normalized $\mathrm{CBV} ; \mathrm{rCBF}=$ relative $\mathrm{CBF} ; \mathrm{rCBV}=$ relative $\mathrm{CBV}$

G lioblastoma (GBM) is the most common primary malignant brain tumor in adults. Surgical tumor resection followed by radiation therapy and concurrent chemotherapy with temozolomide is the current standard therapy for patients with GBM. ${ }^{1}$ Despite multiple treatment approaches, however, the prognosis for patients with GBM is still extremely dismal. ${ }^{2,3}$

GBMs are highly vascularized tumors and have been seen as attractive targets for antiangiogenic therapies. ${ }^{4}$ In particular, vascular endothelial growth factor has been identified as a critical

Received September 25, 2015; accepted after revision March 4, 2016.

From the Department of Radiology (T.J.Y., H.R.C., S.H.C., H.K., S.-W.P., J.-h.K., C.-H.S., M.H.H.), Seoul National University College of Medicine, Republic of Korea; Departments of Radiology (T.J.Y., H.R.C., S.H.C., H.K., J.-h.K., C.-H.S., M.H.H.), Pathology (J.-K.W.), and Neurosurgery (M.H.H.), Seoul National University Hospital, Seoul, Republic of Korea; and Department of Radiology (S.-W.P.), Seoul National University Boramae Medical Center, Seoul, Republic of Korea.

This study was supported by a grant from the National Research Foundation of Korea (NRF-2013RIA1A2008332), Seoul National University Hospital Research Fund (04-2012-1000), Seoul National University College of Medicine Research Fund (800-20140025), and the Institute for Basic Science (IBS-R006-DI) in Korea. regulator of angiogenesis. Bevacizumab is a recombinant humanized monoclonal antibody that binds to human vascular endothelial growth factor and inhibits angiogenesis. ${ }^{5,6}$ It received accelerated FDA approval for treating recurrent GBM in the United States and many other countries and has become the standard of care for treating GBM. ${ }^{5,6}$

DSC perfusion MR imaging can be used as a surrogate marker of perfusion to measure relative $\mathrm{CBV}$ ( $\mathrm{rCBV}$ ) and relative $\mathrm{CBF}$ (rCBF) of patients with GBM. ${ }^{7-10}$ DSC perfusion MR imaging has shown potential as an imaging biomarker to evaluate the antiangiogenic treatment in human patients with GBM..$^{8-11}$ Some re-

Please address correspondence to Seung Hong Choi, MD, PhD, Department of Radiology, Seoul National University College of Medicine, 28, Yongon-dong, Chongno-gu, Seoul, 110-744, Korea; e-mail: choiseunghong@gmail.com

-- Indicates open access to non-subscribers at www.ajnr.org

$\equiv$ indicates article with supplemental on-line tables.

Indicates article with supplemental on-line photo

http://dx.doi.org/10.3174/ajnr.A4800 
searchers have investigated the change in $\mathrm{rCBV}$ or $\mathrm{rCBF}$ in animal GBM models and found significant reduction in animals treated with bevacizumab compared with controls. ${ }^{12,13}$ However, the correlation between perfusion parameters based on DSC perfusion MR imaging and microvascular environment in histologic specimens in subjects treated with antiangiogenic therapy has not been elucidated, to our knowledge.

Arterial spin-labeling (ASL) is a promising perfusion MR imaging technique without using exogenous gadolinium-based contrast agent for CBF quantification, in which the spin population in arterial water magnetically labeled by inversion is used as an endogenous diffusible tracer. ${ }^{14,15}$ Recently, it has been suggested that ASL has the potential to evaluate the response to antiangiogenic therapy in patients with recurrent GBM receiving bevacizumab. ${ }^{16}$

Therefore, the objective of this study was to evaluate the antiangiogenic effect of bevacizumab in a rat GBM model based on ASL perfusion MR imaging relative to a more established technique based on DSC perfusion MR imaging with histopathology.

\section{MATERIALS AND METHODS}

\section{Tumor Cell Line}

Human glioma cells U87 (ATCC, Rockville, Maryland) were cultured at $37^{\circ} \mathrm{C}$ in a humidified $\mathrm{CO}_{2}$ incubator in RPMI $1640 \mathrm{Me}-$ dium (Sigma Aldrich, St. Louis, Missouri), supplemented with $10 \%$ fetal bovine serum.

\section{Animal Model}

This study was approved by the Institutional Animal Care and Use Committee in Seoul National University Hospital (\#12-0238$\mathrm{C} 1 \mathrm{~A} 0$ ) and was performed in accordance with institutional guidelines. Nude athymic rats (200-250 g; Koatec, Gyeonggi-do, Korea) were anesthetized by intraperitoneal injection of a mixture of zolazepam and xylazine, and they were placed in a stereotaxic device. A burr-hole (2-mm-wide) was made on the right side 3 $\mathrm{mm}$ lateral to the midline and $2 \mathrm{~mm}$ proximal to the bregma by using a dental drill. Nude rats were inoculated with U87 glioma cells $\left(3 \times 10^{6}\right.$ cells per $3 \mu \mathrm{L}$ of serum-free RPMI Medium) in the right caudate-putamen region. Cells were injected into the brain by using a Hamilton syringe (Sigma Aldrich) fitted with a 28 gauge needle positioned with a syringe attachment fitted to the stereotaxic device. With stereotaxic guidance, $0,1.4$, and $3.0 \mathrm{~mm}$ were used in areas posterior, lateral, and dorsal to the bregma in the right caudate-putamen, respectively.

Tumor growth was verified by MR imaging at 2 weeks after implantation. Animals were randomly assigned to a control group $(n=4), 3$-day treatment group $(n=6)$, and 10-day treatment group $(n=4)$. Control animals were sacrificed for brain harvest right after the first MR imaging. For the 3-and 10-day treatment groups, bevacizumab was administered intraperitoneally at 20 $\mathrm{mg} / \mathrm{kg}$ in saline right after the first MR imaging. ${ }^{17}$ After 3 days, animals underwent the second MR imaging. Animals in the 3-day treatment group were sacrificed right after the second MR imaging. At 7 days after the second MR imaging, animals in the 10-day treatment group underwent the third MR imaging. They were sacrificed right after the third MR imaging. In addition, to exclude the possible effect of the tumor-volume change on the perfusion parameters, DSC perfusion MR imaging was performed at 0, 3, and 10 days in 4 rats in whom bevacizumab was not injected (10-day-without-treatment group).

The in vivo experimental design of the present study is shown in Fig 1.

\section{MR Imaging Acquisition}

All MR image acquisitions were performed by 2 authors (H.K. with $>10$ years' experience in neuroimaging and T.J.Y. with $>10$ years' experience in neuroradiology) by using a 9.4T MR imaging scanner (Agilent 9.4T/160AS; Agilent Technologies, Santa Clara, California). A volume coil for radiofrequency transmission and a phased array 4-channel surface coil for signal reception (Agilent Technologies) were used for the control, 3-day treatment, and 10-day treatment groups; a single-channel surface coil was used for radiofrequency transmission; and a signal reception (loop coil) (Agilent Technologies) was used for the 10-day-withouttreatment group.

Before MR imaging, animals were anesthetized with 1.5\% isoflurane in room air and placed inside a magnet. Animals were physiologically monitored throughout the MR imaging experiments. To avoid potential changes in cerebral blood perfusion during data collection, we carefully maintained their respiration at $\sim 40$ beats per minute.

Following the acquisition of routine scout images in all 3 orthogonal directions, an automatic shimming procedure was performed. Unenhanced anatomic T2-weighted images were collected by using a fast spin-echo sequence with the following

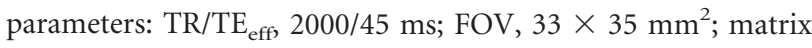
size, $256 \times 256$; echo-train length, 4 ; 15 sections without gap; section thickness, $1 \mathrm{~mm}$; 1 signal average; 2 dummy scans; and receiver bandwidth, $100 \mathrm{kHz}$.

For perfusion data acquisitions by using the ASL technique, an amplitude-modulated pseudocontinuous arterial spin-labeling sequence was used with a single-shot spin-echo echo-planar imaging readout (mCASL; Agilent Technologies). ${ }^{18}$ Vascular and fat-suppression modules were also used with a labeling pulse duration of 3 seconds. Postlabeling delay and the gap between the labeling plane and the central imaging plane were 300 and $20 \mathrm{~mm}$, respectively. Other sequence parameters were the following: TR/ TE, 4000/28 ms; FOV, $33 \times 35 \mathrm{~mm}^{2}$; matrix size, $64 \times 64 ; 3$ sections; section thickness, $2 \mathrm{~mm}$; bandwidth, $250 \mathrm{kHz}$; 60 repetitions for labeled and control data. For quantitative analysis of cerebral blood perfusion, T1 mapping was performed by using a fat-suppressed, single-shot, inversion-recovery spin-echo echoplanar imaging sequence with TR, $8000 \mathrm{~ms}$ and TIs of 15, 35, 80, $200,450,1000,2300$, or $5400 \mathrm{~ms}$. The rest of the sequence parameters were identical to those used for pseudocontinuous ASL data acquisition. ${ }^{18}$ The typical total scan time of ASL was $\sim 10$ minutes.

Finally, DSC images were acquired from the same imaging planes as used for the pseudocontinuous ASL experiments by using a gradient-echo pulse sequence. The sequence parameters were the following: TR/TE, $25 / 5 \mathrm{~ms}$; flip angle, $10^{\circ}$; matrix size, $128 \times 96$; 4 signal averages; bandwidth, $100 \mathrm{kHz} ; 70$ repetitions. After an initial 30-second baseline acquisition, a bolus of gadoterate meglumine $(0.1 \mathrm{mmol}$ per kilogram of body weight; Dotarem; Guerbet, Aulnay-sous-Bois, France) was administered to the 


\section{Control \\ $(n=4)$ \\ 3-days treatment \\ $(n=6)$}

\section{0-days treatment}

( $n=4)$
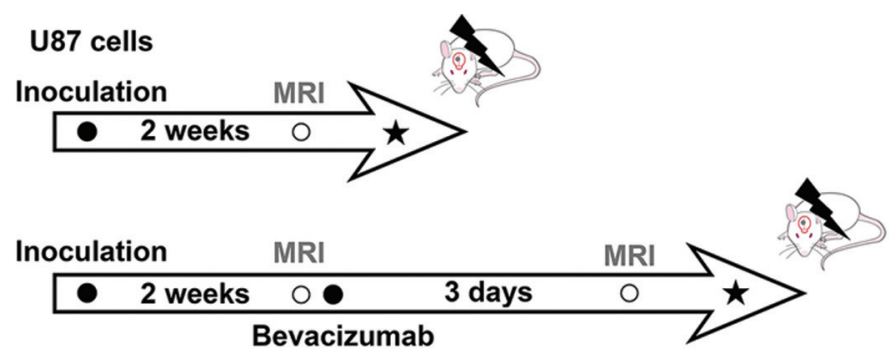

Bevacizumab
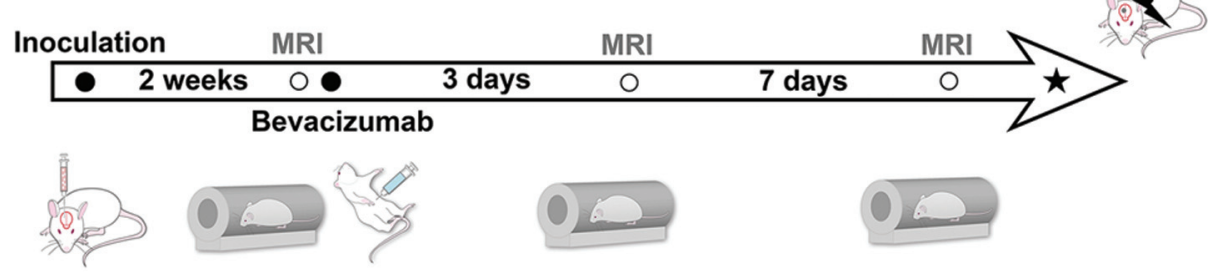

10-days without treatment

$$
(n=4)
$$
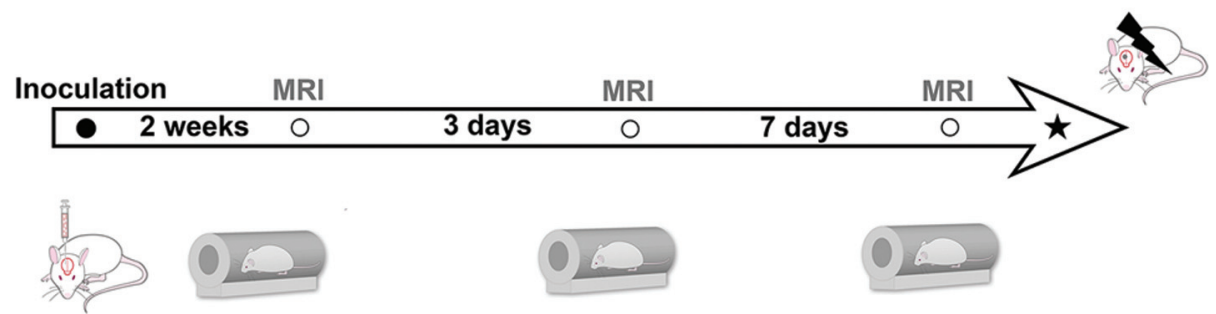

FIG 1. Experimental design showing the timeline of each group for U87 glioma cell inoculation, bevacizumab therapy, MR imaging, and sacrifice for brain harvest.

animals via a tail vein catheter by using a syringe pump $(1 \mathrm{~mL} /$ min; Harvard Apparatus, Holliston, Massachusetts), which was immediately followed by a $1-\mathrm{mL}$ saline flush. The typical total scan time of DSC was $\sim 11$ minutes.

\section{MR Imaging Data Analysis}

All pseudocontinuous ASL images were analyzed by using Matlab (MathWorks, Natick, Massachusetts). CBF maps were derived according to previous reports. ${ }^{18,19}$ A 3-parameter fit was used for estimating T1 maps. Once T1 maps and control and labeled images were obtained, tissue blood flow images were formed according to the following formula: $\mathrm{CBF}=(\lambda / \mathrm{T} 1) \times\left(S_{\text {control }}-\right.$ $\left.S_{\text {label }}\right) /\left(2 \alpha \times S_{\text {control }}\right)$, where $\lambda=0.9$ was the tissue/blood partition coefficient for water, ${ }^{19} S_{\text {control }}$ was the control image signal intensity, $S_{\text {label }}$ was the labeled image signal intensity, T1 was the T1 map, and $\alpha$ was 0.63 as the degree of labeling efficiency. ${ }^{18}$

For DSC perfusion MR imaging, additional preprocessing was performed by using commercial software (nordicICE; NordicNeuroLab, Bergen, Norway), in which T2WIs were used for structural imaging. rCBV maps were generated by using established tracer kinetic models applied to first-pass data. ${ }^{20,21}$ To reduce the effect of recirculation, we fitted $\Delta \mathrm{R} 2^{*}\left(1 / \mathrm{T} 2^{*}\right)$ curves to the $\gamma$-variate function, which was an approximation of the first-pass response as it would appear in the absence of recirculation or leakage. The dynamic curves were mathematically corrected to reduce contrast agent leakage effects. ${ }^{22}$

T2WI, rCBV, and rCBF maps based on DSC, and CBF maps based on ASL were analyzed by using a commercial PACS console. One investigator (T.J.Y. with $>10$ years' experience in neuroradi- ology) who was blinded to the experimental data drew ROIs containing the entire tumor in the plane in which the tumor area was the largest. Tumor boundaries were defined with reference to high-signal-intensity areas thought to represent tumor tissue on the T2WI. ROIs were copied and placed on coregistered $\mathrm{rCBV}$ and rCBF maps based on the DSC and CBF maps from ASL. The mean $\mathrm{rCBV}$ and mean $\mathrm{rCBF}$ of each tumor were measured on rCBV and rCBF maps. The mean rCBV and mean $\mathrm{rCBF}$ of the contralateral hemispheres were also measured as reference values for perfusion parameters. Normalized CBV (nCBV) and normalized $\mathrm{CBF}$ (nCBF) were derived by using the following formulas: $\mathrm{nCBV}=\mathrm{rCBV}_{\text {tumor }} / \mathrm{rCBV}_{\text {reference }}$ and $\mathrm{nCBF}=$ $\mathrm{rCBF}_{\text {tumor }} / \mathrm{rCBF}_{\text {reference }}(\mathrm{On}$-line Fig 1$)$.

\section{Histologic Analysis}

All rats were euthanized in a $\mathrm{CO}_{2}$ chamber soon after MR imaging. Coronal sections sampled across the center of the tumors were fixed in $10 \%$ buffered formaldehyde solution and paraffinembedded. Coronal paraffin sections were used for histologic hematoxylin-eosin staining and immunohistochemical analysis. Histologic evaluation was performed by a pathologist (J.-K.W. with $>10$ years' experience in neuropathology) by using a standard light microscope. Microvessel area (MVA) was determined on the basis of CD34 immunostains. Briefly, areas with the highest microvascular attenuation on CD34-stained sections were identified around the center of the tumor at scanning power. After that, MVAs consisting of endothelial area and vessel lumen were quantified with a higher power $(\times 200$ field $)$ in the selected area. 
Table 1: Comparison of volume and perfusion parameters based on DSC and ASL MR imaging in all tumors ${ }^{\mathrm{a}}$

\begin{tabular}{|c|c|c|c|c|c|c|c|c|c|}
\hline \multirow[b]{2}{*}{ Variables } & \multirow[b]{2}{*}{0 Days $(n=14)$} & \multirow[b]{2}{*}{3 Days $(n=10)$} & \multirow[b]{2}{*}{10 Days $(n=4)$} & \multicolumn{3}{|c|}{ Paired $t$ Test } & \multicolumn{3}{|c|}{ Unpaired $t$ Test } \\
\hline & & & & $P$ Value $^{\mathrm{b}}$ & $P$ Value $^{c}$ & $P$ Value $^{\mathrm{d}}$ & $P$ Value ${ }^{b}$ & $P$ Value ${ }^{c}$ & $\overline{P \text { Value }}$ d \\
\hline Volume $\left(\mathrm{mm}^{3}\right)$ & $40.0(32.3-61.8)$ & 91.9 (53.9-124.1) & 203.7 (103.0-301.3) & .015 & .200 & .117 & .002 & .090 & .086 \\
\hline $\mathrm{nCBV}_{\mathrm{DSC}}$ & $5.9(5.7-6.3)$ & $2.7(2.4-2.9)$ & $0.8(0.6-1.4)$ & $<.001$ & .004 & $<.001$ & $<.001$ & .002 & .005 \\
\hline $\mathrm{nCBF}_{\mathrm{DSC}}$ & $3.7(3.2-4.0)$ & $1.8(1.4-2.2)$ & $0.7(0.6-1.0)$ & $<.001$ & .020 & $<.001$ & $<.001$ & .075 & .004 \\
\hline $\mathrm{nCBF}_{\mathrm{ASL}}$ & $1.7(1.2-2.0)$ & $0.3(0.2-0.5)$ & $0.1(0.1-0.1)$ & $<.001$ & .041 & $<.001$ & .004 & .029 & .050 \\
\hline
\end{tabular}

Note: - $n C B V_{D S C}$ indicates $n C B V$ based on DSC; $n C B F_{D S C}, n C B F$ based on DSC; $n_{C B F}{ }_{A S L}, n C B F$ based on ASL.

a Values are presented as medians (interquartile range, range from the 25 th to the 75 th percentile).

${ }^{\mathrm{b}}$ Results between 0 and 3 days.

c Results between 3 and 10 days.

${ }^{\mathrm{d}}$ Results between 0 and 10 days.

T2WI

0 days
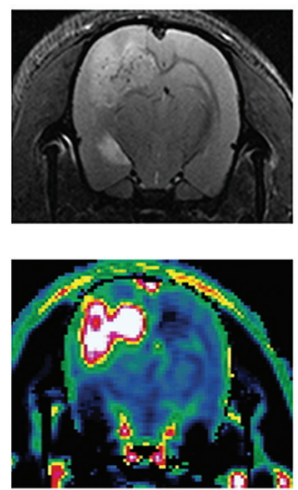

rCBVDSC

rCBFDSC
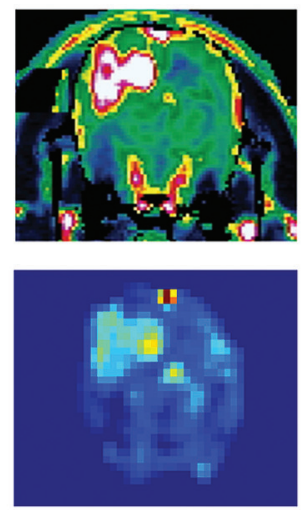

3 days
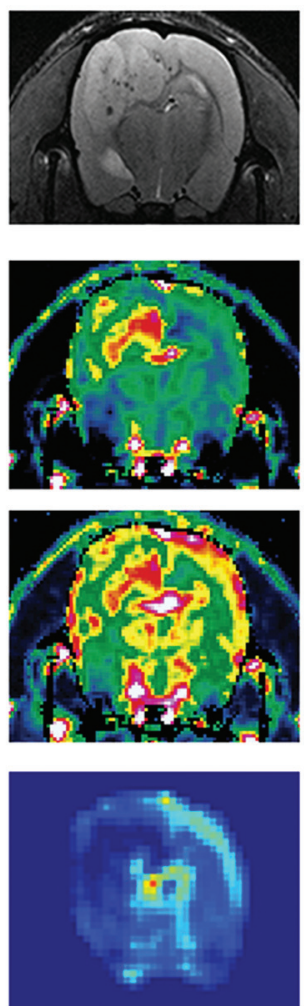

10 days
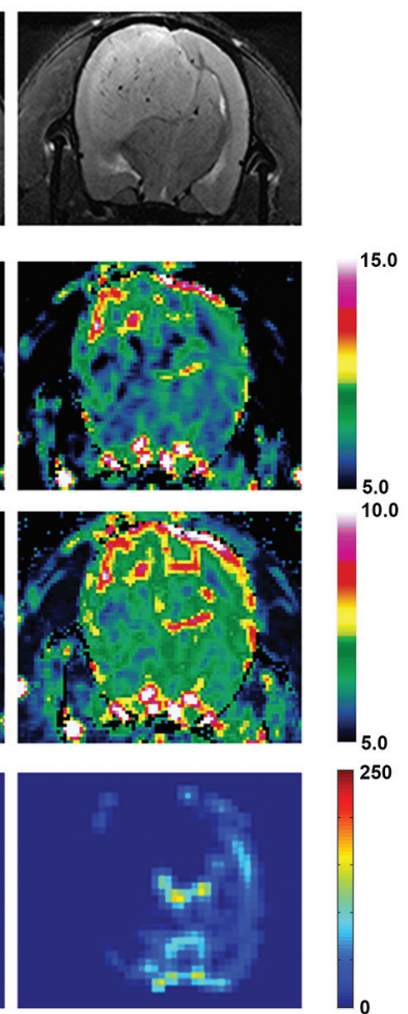
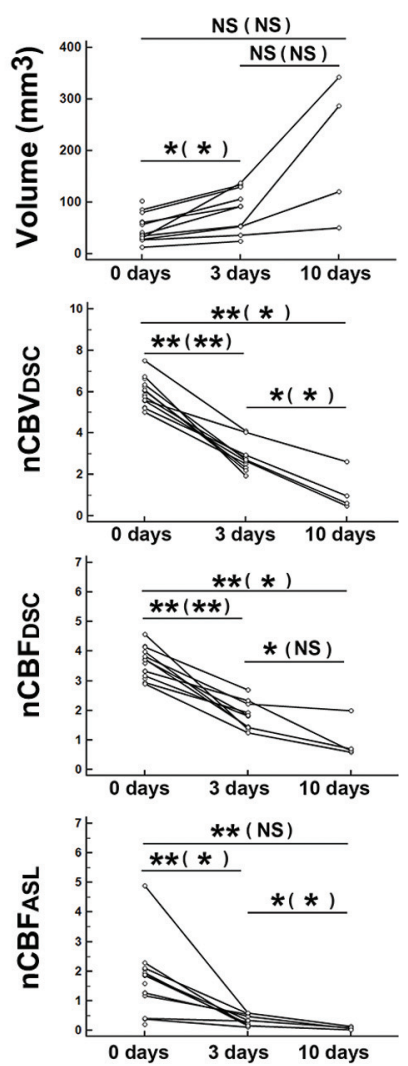

FIG 2. Quantification of tumor volume and perfusion parameters in all tumors. T2WI and perfusion maps were acquired from a rat belonging to the 10-day treatment group. Serial reductions in rCBV and rCBF based on DSC and CBF based on ASL are shown. Graphs in the right column show serial changes of tumor volume and perfusion parameters in all tumors. Scale units of $\mathrm{rCBV}_{\mathrm{DSC}}, \mathrm{rCBF}_{\mathrm{DSC}}$, and $\mathrm{CBF}_{\mathrm{ASL}}$ are milliliters $\times 100 \mathrm{~g} \mathrm{~g}^{-1}$, milliliters $\times 100 \mathrm{~g}^{-1} \times \mathrm{min}^{-1}$, and milliliters $\times 100 \mathrm{~g}^{-1} \times \mathrm{min}^{-1}$, respectively. Data are mean results from paired $t$ tests (unpaired $t$ tests). One asterisk indicates $P<.05 ; 2$ asterisks, $P<.001$; NS, not significant; $\mathrm{nCBV}_{\mathrm{DSC}}, \mathrm{nCBV}$ based on DSC; $\mathrm{nCBF}_{\mathrm{DSC}}, \mathrm{nCBF}$ based on DSC; $\mathrm{nCBF}_{\mathrm{ASL}}, \mathrm{nCBF}$ based on ASL.

Results were expressed as the ratio of the area of microvessel to the total area of analysis within any single $\times 200$ microscopic field.

\section{Statistical Analysis}

Paired and unpaired $t$ tests were used to compare parameters on perfusion MR imaging and MVA on histology within subjects and between groups. For tumors for which histology was available, 1-way analysis of variance with a Scheffe post hoc test was used to analyze differences. Pearson correlation analysis was performed to determine the correlation among perfusion parameters of perfusion MR imaging and MVA. Statistical analysis was performed with the commercially available software: SPSS (Version 12.0 for Windows; IBM, Armonk, New York) and MedCalc for Windows
(Version 9.3.0.0; MedCalc Software, Mariakerke, Belgium). Statistical significance was $P<.05$.

\section{RESULTS}

\section{Analysis of Tumor Volume and Perfusion Parameters} based on DSC or ASL Perfusion MR Imaging in All Tumors

The changes of tumor volume and perfusion parameters in all tumors before and after bevacizumab treatment are summarized in On-line Table 1 and Table 1. In addition, representative images are shown in Fig 2.

After bevacizumab treatment, a tendency of consistent increase in tumor volume was revealed on an unpaired $t$ test $(P<.1)$.

The nCBV of tumors based on DSC showed significant serial 
Table 2: Comparison and correlation of perfusion parameters based on DSC and ASL MR imaging with MVA in tumors with available histology ${ }^{\mathrm{a}}$

\begin{tabular}{|c|c|c|c|c|c|}
\hline Variables & Control $(n=4)$ & $\begin{array}{l}\text { 3-Day Treatment } \\
(n=6)\end{array}$ & $\begin{array}{l}\text { 10-Day Treatment } \\
(n=4)\end{array}$ & $P$ Value $^{\mathrm{b}}$ & Different Groups ${ }^{c}$ \\
\hline Volume $\left(\mathrm{mm}^{3}\right)$ & $51.9(40.8-72.1)$ & $99.7(91.8-124.1)$ & $203.7(103.0-301.3)$ & 0.066 & \\
\hline$n C B V_{D S C}$ & $6.0(5.7-6.3)$ & $2.4(2.2-2.7)$ & $0.8(0.6-1.4)$ & $<.001$ & $\begin{array}{l}\text { (Control) ( } 3 \text { days) ( } 3 \text { days) (10 days) } \\
\text { (Control) (10 days) }\end{array}$ \\
\hline $\mathrm{nCBF}_{\mathrm{DSC}}$ & $3.5(3.3-3.7)$ & $1.8(1.5-1.9)$ & $0.7(0.6-1.0)$ & $<.001$ & (Control) (3 days) (Control) (10 days) \\
\hline $\mathrm{nCBF}_{\mathrm{ASL}}$ & $1.4(1.0-1.7)$ & $0.3(0.2-0.5)$ & $0.1(0.1-0.1)$ & 0.005 & (Control) (3 days) (Control) (10 days) \\
\hline $\operatorname{MVA}\left(\times 10^{-2}\right)$ & $7.5(6.9-7.9)$ & $2.8(2.3-3.3)$ & $2.4(1.9-2.9)$ & $<.001$ & (Control) (3 days) (Control) (10 days) \\
\hline
\end{tabular}

a $V$ alues are presented as medians (interquartile range, range from the 25 th to the 75 th percentile).

Results from 1-way analyses of variances.

'Results from Scheffe post hoc multiple comparisons.

decrease in values after treatment $(P<.05)$. In addition, the $\mathrm{nCBF}$ values based on DSC also showed significant serial decreases in values $(P<.05)$ after treatment, except the results from the unpaired $t$ test between 3 and 10 days $(P=.075)$.

The nCBF values based on ASL showed significant serial decreases $(P<.05)$ after treatment, except the results from the unpaired $t$ test between 0 and 10 days $(P=.050)$.

\section{Analysis of Tumor Volume and Perfusion Parameters based on DSC and ASL Perfusion MR Imaging and MVA in Tumors with Available Histology}

Comparison and correlation of tumor volume and perfusion parameters from DSC and ASL perfusion MR imaging and MVA in tumors with available histology are summarized in Table 2. In addition, representative images are shown in Fig 3.

There was a tendency toward an increase in tumor volume with $P<.1(P=.066)$.

All the nCBV from DSC, nCBF from DSC, nCBF from ASL, and MVA values showed significant decreases after treatment $(P<.001, P<.001, P=.005$, and $P<.001$, respectively). The Scheffe post hoc multiple comparisons revealed significant $(P<.05)$ differences, except between the 3-and the 10-day treatment groups for $\mathrm{nCBF}$ from DSC, nCBF from ASL, and MVA values.

The nCBV and nCBF based on DSC and nCBF based on ASL significantly correlated with MVA (correlation coefficient, $r=$ $0.911,0.869$, and 0.860 , respectively; $P<.001$ for all). nCBF from ASL perfusion MR imaging also had a positive and significant correlation with $\mathrm{nCBF}$ and $\mathrm{nCBV}$ based on DSC MR imaging $(r=$ 0.8298 and 0.789 , respectively; $P<.001$ for both) (On-line Fig 2 ). However, nCBF (median [interquartile range], 0.2 [0.1-0.6]) measured on ASL perfusion MR imaging was significantly less than nCBF $(1.9[1.4-3.0])$ based on DSC MR imaging $(P<.001)$.

\section{Analysis of Tumor Volume and Perfusion Parameters based on DSC in the 10-Days-without-Treatment Group}

The changes of tumor volume and perfusion parameters in tumors without antiangiogenic treatment are summarized in Online Table 2.

At 10 days after the first MR imaging, the volume of tumors increased with significant differences between any days $(P$ values between 0 and 3 days, 3 and 10 days, and 0 and 10 days, .041, .013, and $<.001$, respectively).

The nCBV of tumors in the 10-day group was significantly higher than that in the 0 -day group ( $P$ values between 0 and 3 days, 3 and 10 days, and 0 and 10 days, .034, .067, and .040, re- spectively). The nCBF of tumors in the 10-day group was not significantly higher than that in the 0 -day group ( $P$ values between 0 and 3 days, 3 and 10 days, and 0 and 10 days, .156, .055, and .070, respectively) (On-line Fig 3 ).

\section{DISCUSSION}

In the present study, we assessed the changes of perfusion parameters in the GBM rat model treated with the antiangiogenic agent bevacizumab based on DSC and ASL perfusion MR imaging. We found that perfusion parameters based on ASL and DSC perfusion MR imaging decreased after antiangiogenic treatment, which correlated well with histopathology results such as MVA.

Because bevacizumab has been reported to have an antiangiogenic effect in GBM and is approved as a treatment agent either alone or in combination with chemotherapy, ${ }^{5,23}$ the potential of perfusion MR imaging to precisely characterize the microvascular environment is clinically relevant. However, it was unclear whether perfusion MR imaging can be applied as an imaging biomarker to quantify the microvascular environment mainly due to significant intra- and intertumoral biologic heterogeneity of GBM. Although some researchers have investigated the potential of DSC perfusion MR imaging in GBMs treated with bevacizumab in animal models, ${ }^{12,24}$ direct correlation between perfusion parameters and histologic features was not elucidated. In terms of the ASL perfusion MR imaging technique, a previous report has suggested the potential of using ASL perfusion MR imaging to evaluate the response to antiangiogenic therapy in a patient with recurrent GBM who received bevacizumab. ${ }^{16}$ However, additional investigation in human studies or animal models has not been elucidated, to our knowledge.

According to our results, $\mathrm{nCBV}$ and $\mathrm{nCBF}$ values based on DSC showed significant serial reduction after bevacizumab treatment with a strong correlation with MVA in histology. Thus, they have the potential as imaging biomarkers to evaluate the antiangiogenic effect in GBM treated with bevacizumab. In addition, our results revealed that $\mathrm{nCBF}$ values based on the ASL technique have significant serial reduction after bevacizumab treatment with a strong correlation with MVA.

The measurement of hemodynamic parameters such as CBV and CBF based on DSC perfusion MR imaging can be compromised by elevated vascular permeability and leakage of contrast agent, both of which can be heavily influenced by the biologic changes in GBM under treatment. ${ }^{25}$ However, the ASL perfusion MR imaging technique uses endogenous contrast agents without the need to inject contrast agent. Therefore, the ASL perfusion 


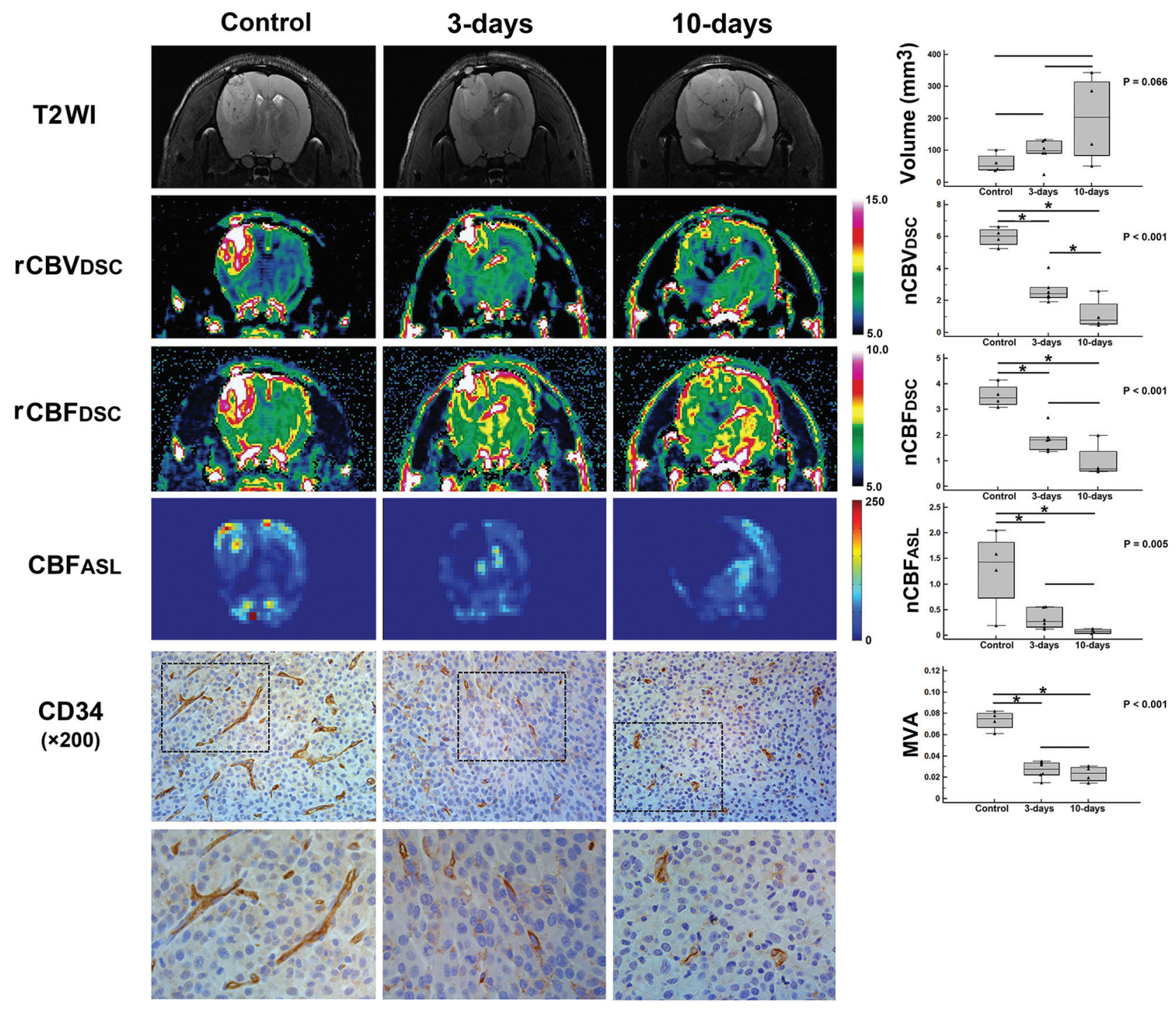

FIG 3. Quantification of tumor volume, perfusion parameters, and MVA in tumors with available histology. T2WI and perfusion maps were acquired from rats in the control group, 3-day treatment group, or 10-day treatment group. Differences in rCBV and rCBF based on DSC, CBF based on ASL, and MVA are shown. Graphs in the right column show differences in the tumor volume and hemodynamic parameters and MVA. In the lower images, the tumors stained immunohistochemically with anti-CD34 show positive brown cytoplasmic staining of the endothelial area and vessel lumen. Scale units of $\mathrm{rCBV}_{\mathrm{DSC}}, \mathrm{rCBF}_{\mathrm{DSC}}$, and $\mathrm{CBF}_{\mathrm{ASL}}$ are milliliters $\times 100 \mathrm{~g}^{-1}$, milliliters $\times 100 \mathrm{~g}^{-1} \times \mathrm{min}^{-1}$, and milliliters $\times 100 \mathrm{~g}^{-1}$ $\times$ min $^{-1}$, respectively. $P$ values were based on 1-way analyses of variances. One asterisk indicates a significant $(P<.05)$ difference from the Scheffe post hoc multiple comparisons.

MR imaging technique might overcome the drawbacks of DSC perfusion MR imaging. In addition, ASL perfusion MR imaging techniques can make it feasible to characterize the microvascular environment and to evaluate the antiangiogenic effect against GBM in patients who have contraindications to contrast agent administration.

However, nCBF values based on ASL perfusion MR imaging had a significantly lower value compared with those based on DSC. Leakage of contrast agent on DSC and insufficient labeling efficacy due to unestablished postlabeling decay time in animal models may contribute to the difference in $\mathrm{nCBF}$ values based on DSC and ASL. Because the appropriate postlabeling decay time has been established for brain imaging in humans, such discrepancies may be minimized in the application of ASL perfusion MR imaging for human GBM. Even though nCBV values based on DSC perfusion MR imaging had significant differences in animals between the 3-day and 10-day-treatment groups, no significant difference in the MVA values was found between the 2 groups. Therefore, perfusion parameters based on DSC perfusion MR imaging might have been influenced by other factors related to the antiangiogenic mechanism beyond MVA.

Most interesting, the present study revealed a tendency of consistent increase in tumor volume despite reduced $\mathrm{nCBV}$ and nCBF values $(P<0.1)$. Although we used $20 \mathrm{mg} / \mathrm{kg}$ of bevacizumab in this study based on a previous report that investigated its dose-dependent effect on GBM blood vessels, ${ }^{17}$ the tumor volume showed a serial increase in all tumors (even though the growth rate seems to be lower than that in the 10-day-withouttreatment group). Our result is consistent with those in previous reports, ${ }^{12,17}$ suggesting a possible insufficient effect of antiangiogenic therapy on tumor proliferation and/or a possible evasion mechanism against the antiangiogenic drug in GBM.

AJNR Am J Neuroradiol 37:1650-56 Sep 2016 www.ajnr.org 


\section{CONCLUSIONS}

We demonstrated that CBF values based on ASL and CBV and CBF values based on DSC have good performance for evaluating antiangiogenic therapy, with strong correlations with MVA in a rat GBM model. ASL perfusion MR imaging has the potential to be used as a noninvasive imaging biomarker to monitor the effect of antiangiogenic therapy for GBM.

Disclosures: Tae Jin Yun, Seung Hong Choi-RELATED: Grant: government fund.* Moon Hee Han-UNRELATED: Consultancy: MicroVention. *Money paid to the institution.

\section{REFERENCES}

1. Stupp R, Mason WP, van den Bent MJ, et al; European Organisation for Research and Treatment of Cancer Brain Tumor and Radiotherapy Groups, National Cancer Institute of Canada Clinical Trials Group. Radiotherapy plus concomitant and adjuvant temozolomide for glioblastoma. $N$ Engl J Med 2005;352:987-96 CrossRef Medline

2. Erpolat OP, Akmansu M, Goksel F, et al. Outcome of newly diagnosed glioblastoma patients treated by radiotherapy plus concomitant and adjuvant temozolomide: a long-term analysis. Tumori 2009;95:191-97 Medline

3. Jeon HJ, Kong DS, Park KB, et al. Clinical outcome of concomitant chemoradiotherapy followed by adjuvant temozolomide therapy for glioblastaomas: single-center experience. Clin Neurol Neurosurg 2009;111:679-82 CrossRef Medline

4. Jain RK, di Tomaso E, Duda DG, et al. Angiogenesis in brain tumours. Nat Rev Neurosci 2007;8:610-22 CrossRef Medline

5. Friedman HS, Prados MD, Wen PY, et al. Bevacizumab alone and in combination with irinotecan in recurrent glioblastoma. J Clin Oncol 2009;27:4733-40 CrossRef Medline

6. Kreisl TN, Kim L, Moore K, et al. Phase II trial of single-agent bevacizumab followed by bevacizumab plus irinotecan at tumor progression in recurrent glioblastoma. J Clin Oncol 2009;27:740-45 CrossRef Medline

7. Artzi M, Blumenthal DT, Bokstein F, et al. Classification of tumor area using combined DCE and DSC MRI in patients with glioblastoma. J Neurooncol 2015;121:349-57 CrossRef Medline

8. Harris RJ, Cloughesy TF, Hardy AJ, et al. MRI perfusion measurements calculated using advanced deconvolution techniques predict survival in recurrent glioblastoma treated with bevacizumab. J Neurooncol 2015;122:497-505 CrossRef Medline

9. Schmainda KM, Prah M, Connelly J, et al. Dynamic-susceptibility contrast agent MRI measures of relative cerebral blood volume predict response to bevacizumab in recurrent high-grade glioma. Neuro Oncol 2014;16:880-88 CrossRef Medline

10. Stadlbauer A, Pichler P, Karl M, et al. Quantification of serial changes in cerebral blood volume and metabolism in patients with recurrent glioblastoma undergoing antiangiogenic therapy. Eur $J$ Radiol 2015;84:1128-36 CrossRef Medline

11. Sawlani RN, Raizer J, Horowitz SW, et al. Glioblastoma: a method for predicting response to antiangiogenic chemotherapy by using MR perfusion imaging-pilot study. Radiology 2010;255:622-28 CrossRef Medline

12. Jalali S, Chung C, Foltz W, et al. MRI biomarkers identify the differential response of glioblastoma multiforme to anti-angiogenic therapy. Neuro Oncol 2014;16:868-79 CrossRef Medline

13. Keunen O, Johansson M, Oudin A, et al. Anti-VEGF treatment reduces blood supply and increases tumor cell invasion in glioblastoma. Proc Natl Acad Sci U S A 2011;108:3749-54 CrossRef Medline

14. Detre JA, Alsop DC. Perfusion magnetic resonance imaging with continuous arterial spin labeling: methods and clinical applications in the central nervous system. Eur J Radiol 1999;30:115-24 CrossRef Medline

15. Detre JA, Leigh JS, Williams DS, et al. Perfusion imaging. Magn Reson Med 1992;23:37-45 CrossRef Medline

16. Fellah S, Girard N, Chinot O, et al. Early evaluation of tumoral response to antiangiogenic therapy by arterial spin labeling perfusion magnetic resonance imaging and susceptibility weighted imaging in a patient with recurrent glioblastoma receiving bevacizumab. J Clin Oncol 2011;29:e308-11 CrossRef Medline

17. von Baumgarten L, Brucker D, Tirniceru A, et al. Bevacizumab has differential and dose-dependent effects on glioma blood vessels and tumor cells. Clin Cancer Res 2011;17:6192-205 CrossRef Medline

18. Utting JF, Thomas DL, Gadian DG, et al. Understanding and optimizing the amplitude modulated control for multiple-slice continuous arterial spin labeling. Magnetic Reson Med 2005;54:594-604 CrossRef Medline

19. Silva AC, Kim SG, Garwood M. Imaging blood flow in brain tumors using arterial spin labeling. Magnetic Reson Med 2000;44:169-73 Medline

20. Maeda M, Itoh S, Kimura H, et al. Tumor vascularity in the brain: evaluation with dynamic susceptibility-contrast MR imaging. $R a$ diology 1993;189:233-38 CrossRef Medline

21. Rollin N, Guyotat J, Streichenberger N, et al. Clinical relevance of diffusion and perfusion magnetic resonance imaging in assessing intra-axial brain tumors. Neuroradiology 2006;48:150-59 CrossRef Medline

22. Aronen HJ, Gazit IE, Louis DN, et al. Cerebral blood volume maps of gliomas: comparison with tumor grade and histologic findings. $R a-$ diology 1994;191:41-51 CrossRef Medline

23. Vredenburgh JJ, Desjardins A, Herndon JE 2nd, et al. Phase II trial of bevacizumab and irinotecan in recurrent malignant glioma. Clini Cancer Res 2007;13:1253-59 CrossRef Medline

24. Keunen $O$, Taxt $T$, Gruner R, et al. Multimodal imaging of gliomas in the context of evolving cellular and molecular therapies. Adv Drug Deliv Rev 2014;76:98-115 CrossRef Medline

25. Essig M, Shiroishi MS, Nguyen TB, et al. Perfusion MRI: the five most frequently asked technical questions. AJR Am J Roentgenol 2013;200:24-34 CrossRef Medline 